\title{
Healing Wounds under Mechanical Stress: A Case Example
}

\author{
Jennifer D. Hastings ${ }^{1}$, Sara Shapiro ${ }^{2}$ \\ ${ }^{1}$ University of Puget Sound School of Physical Therapy, Tacoma, WA, USA \\ ${ }^{2}$ Apex Health Solutions, Tacoma, WA, USA \\ Email: jhastings@pugetsound.edu
}

Received 18 January 2016; accepted 15 February 2016; published 19 February 2016

Copyright (C) 2016 by authors and Scientific Research Publishing Inc.

This work is licensed under the Creative Commons Attribution International License (CC BY).

http://creativecommons.org/licenses/by/4.0/

(c) (i) Open Access

\begin{abstract}
This paper challenges the concept that the essential element in wound healing is to offload pressure. We suggest a change in approach that recognizes the integumentary system as one which, like all other body systems, adapts to the stresses put upon it. We use a clinical case example to illustrate the use of intentional mechanical stress to promote wound healing and include a review of the relevant literature. The intent of this publication is to call for a new look at clinical practice regarding wound healing and to call for future research directed at investigation of this theory.
\end{abstract}

\section{Keywords}

Wounds, Pressure Ulcer, Mechanical Stress, Electric Stimulation, Weight-Bearing

\section{Introduction}

Offloading pressure has been common practice in the treatment of wounds. The assumption that pressure causes wounds is nearly universal, hence the commonly used names are "pressure sore" or "pressure ulcer". The first documented "bed sores" were found in populations who were severely debilitated, and immobile patients with acute brain injury and spinal cord injury (SCI), residing in nursing homes [1]. The wounds appeared on areas where bone was the most subcutaneous and therefore the idea that these were high points of pressure began. Response to the existence of bed sores was the mandate for turning patients on schedules to prevent buildup of pressure. More recent clinical practice guidelines address comorbid issues of malnutrition, depression, dehydration, and incontinence and consider shear as well as pressure, but pressure remains the primary focus of prevention and intervention in wounds in the SCI population [2]. With SCI the prevalence of pressure sores is high. In a recent study, 185 SCI patients who were admitted to a single facility over a six month period were reviewed, with $49.2 \%$ having at least 1 (and sometimes multiple) pressure ulcers [3]. There is also a very high incidence of 
recurrence, with $20 \%$ of surgically repaired wounds recurring within 3 months of surgery [4]. The best predictor of a second pressure ulcer in the SCI population is the presence of a first pressure ulcer [5] [6].

What if we are impairing the tissue healing with off-loading?

We are aware that human tissue is adaptive to stresses put upon it. Thus, in the absence of pathology, bone will become denser with high loading or, muscles can hypertrophy in response to resistance training. Skin has self-protective measures of callous when habitual friction is encountered, and we know with certainty that female skin responds to the stressor of pregnancy with overt elongation of tissue (albeit likely influenced by hormones). Tissues respond to the physical demands of their environment by adapting to the stress. The manipulation of the physical environment can be used therapeutically to stimulate repair.

This paper reports a case where the concept that the skin must heal under the stresses that it must endure, was integral to the wound healing intervention. The second, somewhat unique, feature of this case is that the wound care interventions were provided by family and lay caregivers in the home under the instruction and monitoring by the physical therapist (PT). Houghton found that electrical stimulation (ES) could be administered safely and efficiently by lay caregivers [7] and the finding is supported by this case. Interventions included positioning, dressing changes, soft tissue mobilization, massage, progressive reseating and high volt ES (parameters and electrode placement set by PT). Institutional review board approval was received for this case review and personal permission of the individual involved was granted.

Why should ES and concomitant tissue stress and loading work? The ES is a facilitator of natural healing. The mechanism of healing with ES is not different from the normal healing process. ES attracts specific cells to the area of injury based on the polarity of the cells and their response. Current evidence shows that the specific polarity of the ES is used to draw the appropriate cells to the area of injury. Because the normal polarity of the skin without injury tends to be slightly negative and the current of injury which develops as a result of the insult, the initial treatment of electrical stimulation starts with negative (anodal) polarity.

Wolff's Law indicates that a pulsatile current creates a mechanical force that perturbs cells, causing contraction and expansion that is thought to increase collagen deposition [8]. Electrical stimulation (ES) can be considered as a mechanical stress which is a facilitator of tissue healing [9]. The mechanical perturbation changes in membrane permeability, ion movement across the cell membrane, and increased blood flow and shear force; all seem to contribute to increasing healing. Thus the addition of ES to the normal stress of loading appears to increase not only the rate of repair but the strength of the tissue that is healed.

The motivation for writing this case report is the fact that it has now been 7-year post healing and the individual has had no recurrent breakdown of this wound, nor breakdown of the surrounding tissue even in the presence of multiple challenges to health including 2 lower extremity and one upper extremity fracture which resulted in modified seated positions.

\section{Case Report}

At the time of wound care intervention the individual presented in this case report was a 25-year-old woman with C5 AIS A tetraplegia of 6 years with history of recurrent grade II right ischial pressure ulcer. Concomitant with systemic medical decline (aplastic anemia) the wound declared and progressed to grade IV. Strict bed rest was not possible during early phase of wound care due to need for medical appointments; bed rest was also lifted for attendance at church and for planned social activities and holiday functions. No more than 10 days of bed rest in sequence was ever experienced. Prior to PT involvement, wound care consisted of enzymatic debridement and dressing changes supervised by an outpatient wound clinic. PT promoted healing with intentional tissues stresses. Initially the tissue was stressed with high volt ES, then massage and soft tissue mobilization surrounding wound, then with daily positioning with hip and knees at 90 degrees while in supine, and finally with progressive sitting. Intentional mechanical stress was done for facilitation of healing.

\section{Intervention}

A home visiting PT did wound assessments and established wound care interventions and instructed the patient's mother in how to administer all wound care interventions. At the highest frequency PT visited daily for up to three days in a row (initially and again at week six) but for most of the duration of treatment PT visits were once a week or every other week.

Figure 1 shows the key components and timing of intervention. ES was initially done with direct technique, 


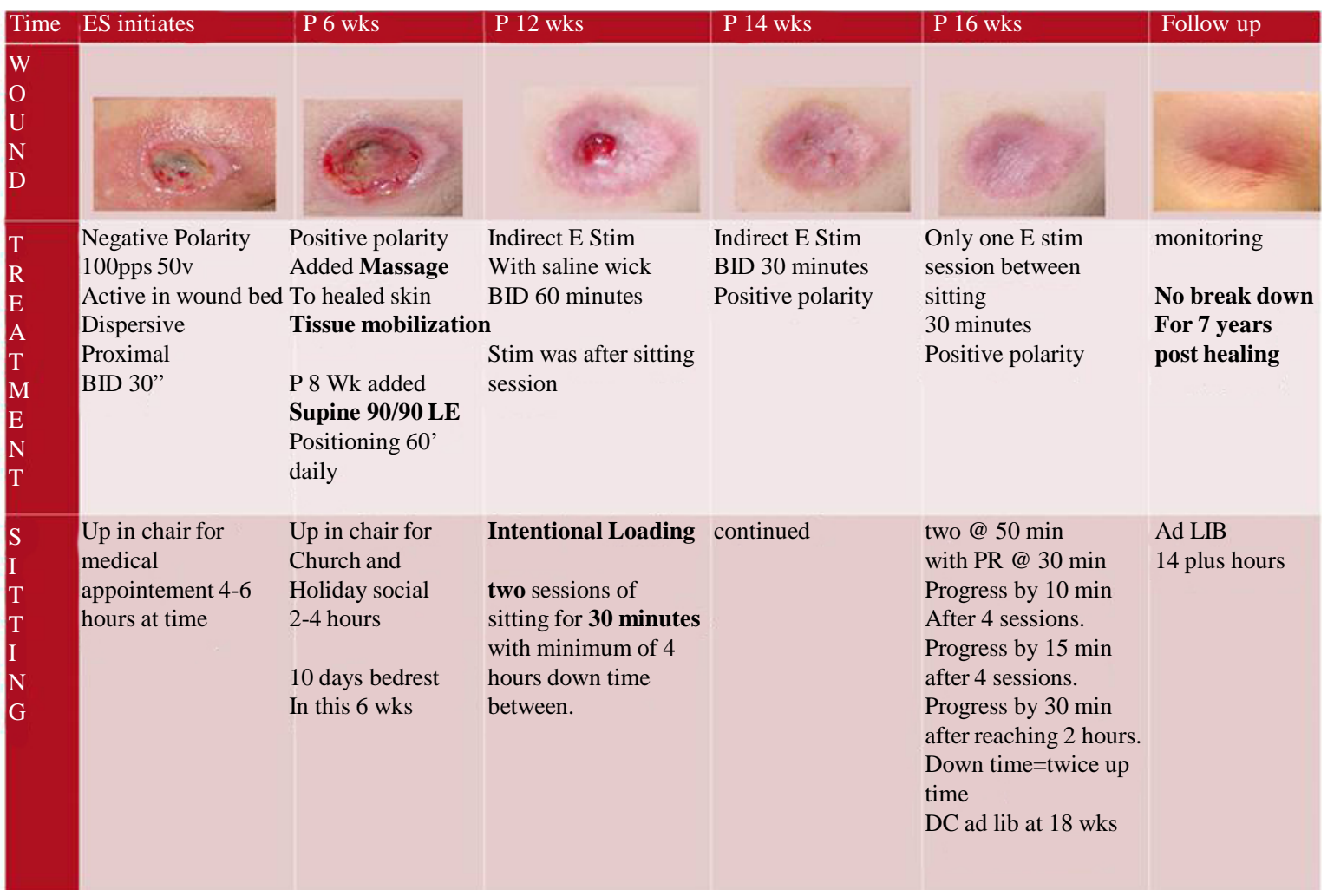

Figure 1. Key aspects of intervention. pps = pulse per second, $v=$ volts, BID = twice a day, $\mathrm{p}=$ post, wk (s) = week (s), $\mathrm{LE}=$ lower extremity, $\min =$ minutes, $A d L I B=$ without restriction.

where the active electrode was in the wound bed with saline soaked gauze as conduit. The polarity of the treating electrode was initially negative, and then changed to positive once wound appeared clean and without odor (after 4 weeks), polarity was changed back to negative for 3 days during week 7 due to a period of wound stagnation, and then held at positive for the duration of treatment. When the wound became very small at the surface but maintained significant depth the technique was changed to indirect ES (electrodes then placed peri-wound) with a saline wick. Indirect ES is when the active electrode is no longer placed in the wound bed. The dispersive is still proximal but two active electrodes are place in the wound periphery, so that the current continues to run through the wound. A saline wick is a saline soaked $1 / 4$ inch cloth dressing lightly packed into the deep wound channel. Additionally the therapist added intentional loading to the intervention plan. Two sessions of sitting for 30 minutes with minimum of 4 hours off loading in bed rest between the sitting sessions were scheduled daily. This continued for 4 weeks until wound closure. The family kept a journal and was highly compliant. Most episodes of sitting were $+/-3$ minutes of the prescribed thirty minutes and there was one episode of only 3.5 hours off loading in the last week. The rationale for the intentional loading was that the wound depth was increasing due to wound contraction and therapist theorized that compression would avoid tract formation. After wound was completely closed (at 16 weeks after initiating ES) the ES treatment continued and seating was progressed intentionally with prescribed time for loading and off-loading with ES between sitting sessions. Progressing to completely ad lib sitting occurred over 2.5 weeks and ES continued during this time. The duration of therapy supervised wound care was 18 weeks.

\section{Results}

Figure 2 and Figure 3 show the healing pattern of the wound as measured in centimeters. Volume is calculated by the measured greatest length times the perpendicular width times the depth.

Full closure was achieved and $a d$ lib sitting for up to 14 hours. More significantly this individual has been followed by one author for seven years and there has not been any recurrence of this wound nor breakdown of adjacent tissues. 


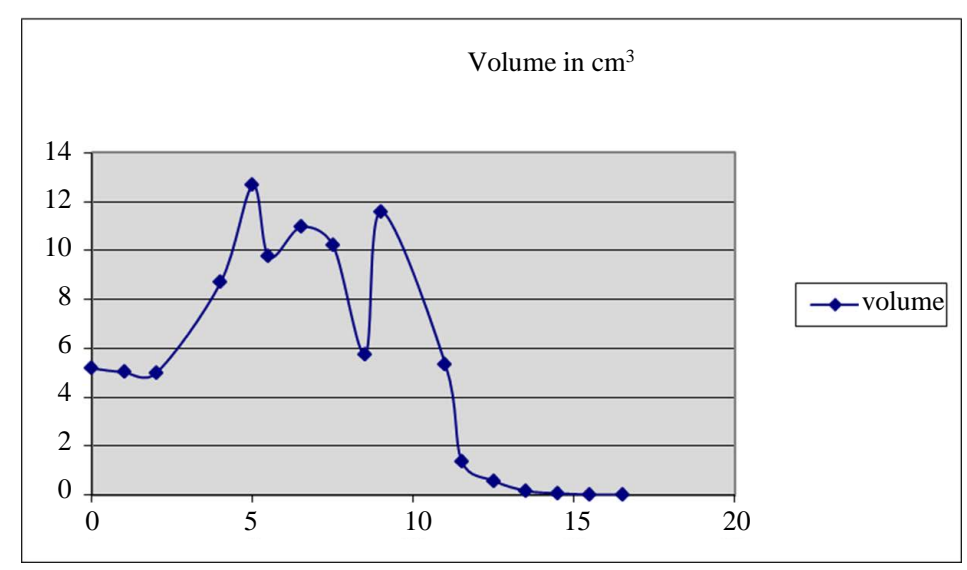

Figure 2. Wound volume over time in weeks.

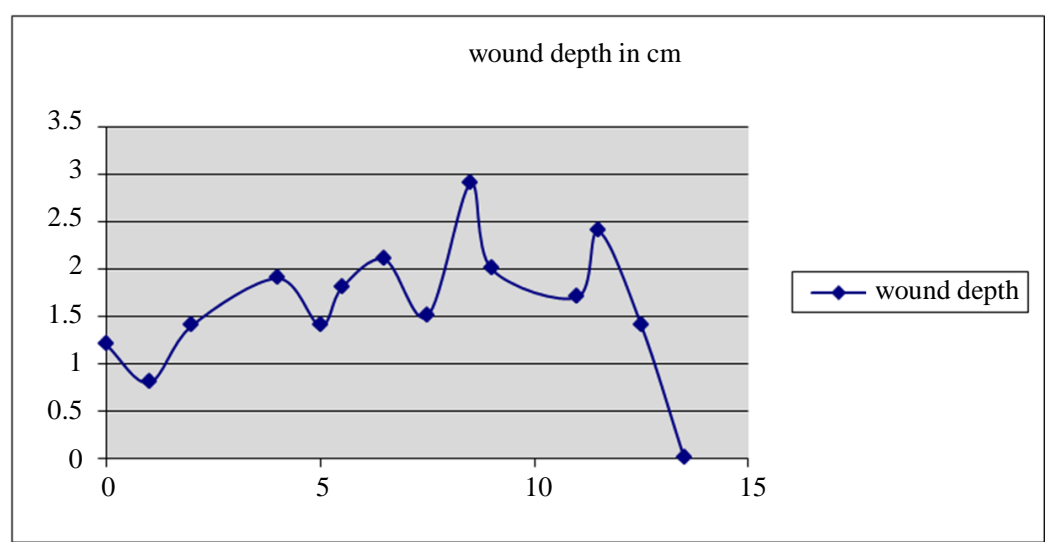

Figure 3. Wound depth over time in weeks.

\section{Discussion}

There is evidence that prolonged unloading can be detrimental. Reperfusion following unloading causes further buildup of damage [10]. There is a rise in damage even after the insult is removed [11]. Pietramaggiori et al. showed that both 4 days of continuous and similar cyclic loading showed a fourfold increase in cellular proliferation in the epidermis and a 2.8 fold increase in circulation [12].

The use of high volt ES to support wound healing has been common practice since 1960s [13]. Evidence supports this practice and the CMS approved payment for ES for wound care in 2002 [14]. Electric currents are designed to mimic the "current of injury". There is a natural and normal current which flows because of the gradient of ion concentration in the cells, each with a different electrical potential. A current of injury is generated when an injured part of a nerve, muscle, or other excitable tissue is injured. The evidence from preclinical studies [15] and clinical studies [16] indicates that healthy skin is characterized by a slightly negative current, while injured tissue is more commonly associated with a positive current. The body generates an electrical potential across these tissues. This potential is active in wound healing by promoting cellular migration. A host of events are triggered by electrical stimulation, which includes angiogenesis, granulation and re-epithelialization. Thus, the use of electrical stimulation is intended to facilitate the normal process of cell migration necessary to move the wound from the inflammatory phase forward and into the proliferation phase. Nonetheless, there is still controversy in the exact electrode configuration, polarity and even waveform offering the best outcome.

Wounds appear to require an extended period of time to heal because the body's endogenous system has been disrupted. There are quite a number of studies- both human and animal- which suggest that the ES may assist in the inflammatory phase by inhibiting inflammation and the growth of micro-organisms [17]. Electrical stimulation is believed to promote tissue healing by attracting appropriate cells to the area of the wound. At that point, the electrical stimulation facilitates altered cell membrane permeability, and an ultimate modification of endo- 
genous electrical potential within the tissue. Specific cells can be attracted to the injured healing area by the addition of this electrical charge, because the cells carry a charge. Cell types such as lymphocytes, leukocytes, macrophages, and fibroblasts as well as activated neutrophils, are present when a wound is inflamed, and attracted to the negative pole [13] [16]. A number of in vivo studies have shown increases in the synthesis of adenosine triphosphate (ATP) and proteins as well [15].

In the second phase of wound healing, the proliferation of collagen production facilitates the granulation process. The application of an electric current stimulates an increase in production of DNA which is used for fibroblast synthesis [18]. The dermis, made up of primarily fibroblasts, is influenced by the electrical current as well. Studies show that fibroblasts migrate toward the anode.In wounds that were actively created and then subjected to electric stimulation, the fibroblasts actively migrated from the wound edges and closed the wound entirely in 24 hours [19] [20]. It appears that the granulation phase is promoted through an increase in collagen production and fibroplasia [21]. With the last phase of wound healing, epithelialization is assisted by electrical stimulation as well. The keratinocytes are in the majority within the cellular population. Keratinocytes are involved in re-epithelialization and migrate toward the cathode.

Why does it matter? Recurrent rates for pressure ulcers are astoundingly high. If tissue can not only be healed with an inexpensive home based intervention but healed BETTER with a stronger and more durable tissue which withstands the usual daily threats, this is a far superior outcome. The costs of this intervention were very low. At the highest frequency the PT provided daily visits, but usually once a week or every other week. There were a total of 16.5 PT hours over 27 visits. The actual interventions were being provided by the trained caregiver using parameters established by the PT. The total cost for PT services: \$2,435 (this service was provided by a direct access cash based private practice under referral from a consulting MD). Charges to the family were for PT travel as well as time in home for PT services. Tele consultation via phone or email was not billed.

Typical costs for surgical repair of pressure ulcers are significantly higher. A British study found the direct medical cost of surgically treating one pressure ulcer to be $€ 20,957$ [22]. In a Canadian study authors found an average monthly cost of \$4745 (CAD) with a median duration of 25 months [23] and for community acquired pressure ulcers a US study determined the cost was \$124,327 with an average of 4 hospital re-admissions [24]. There are more considerations than just the cost of the surgical intervention. Surgery requires hospitalization, and for the spinal cord injured individual who is already immune compromised just being in a hospital environment is a risk. There is research to suggest that there is an increasing risk of iatrogenic infections for populations who are immunosuppressed [25]. Whereas, if the individual can stay at home where they have good social support and physical caregiving the potential for better overall health is higher. The improved psychosocial environment of the home can only be supportive to healing. Moreover, if the individual can maintain their social roles, continuing to work or be a student, this improves overall quality of life.

At a minimum, healed tissue needs to tolerate the normal daily motions of the body. It is the clinical experience of both authors to see surgical skin repair (flap procedures) dehisce, or otherwise fail. In many cases the failure will occur upon re-sitting. If you heal skin of seated surfaces in a position of hip extension it makes intuitive sense that the length of the healed tissue will not be sufficient for hip flexion. This consideration should be taken into three dimensions. The usual sliding, compression and rotational forces experienced by the skin will want to be tolerated.

The evidence supporting the approach used in this case is limited by the lack of high-quality studies, the lack of isolated electrical stimulation intervention groups, and the absence of clinical studies that included wound closure under intentional mechanical or loading stress. Further limitations of research in this area are the heterogeneity in study methodologies small sample size, limited follow up data collection and lack of follow through until complete wound closure.

\section{Conclusion}

Overload is a generative stimulus for every system in the human body. Bones calcify with weight bearing and muscles hypertrophy under resistance load. The cardiopulmonary system responds to overload. The nervous system is now understood to "learn" or reorganize with repetitive problem solving (stress). It should logically hold that skin will respond in the same manner to mechanical stress. We suggest if we intentionally stress the healing tissue and then supplement the healing with a facilitator (ES), this has the theoretical potential to create a more rapid and successful outcome. Research is needed to investigate this theory in larger samples of men and women with SCI. 


\section{References}

[1] Levine, J.M. (2005) Historical Perspective on Pressure Ulcers: The Decubitus Ominosus of Jean-Martin Charcot. Journal of the American Geriatrics Society, 53, 1248-1251. http://dx.doi.org/10.1111/j.1532-5415.2005.53358.x

[2] Consortium of SC Medicine (2000) Pressure Ulcer Prevention and Treatment Following SCI: A Clinical Practice Guideline for Health Care Professionals. Paralyzed Veterans of America, Washington DC.

[3] Scheel-Sailer, A., Wyss, A., Boldt, C., Post, M.W. and Lay, V. (2013) Prevalence, Location, Grade of Pressure Ulcers and Association with Specific Patient Characteristics in Adult Spinal Cord Injury Patients during the Hospital Stay: A Prospective Cohort Study. Spinal Cord, 51, 828-833. http://dx.doi.org/10.1038/sc.2013.91

[4] Chua, W. (2012) Peri-Operative Care for Pressure Ulcer Surgery; Optimizing for Successful Outcomes. Presented at the PVA Summit 2012, Las Vegas, 28 August 2012.

[5] Chen, Y.Y., DeVivo, M.J. and Jackson, A.B. (2005) Pressure Ulcer Prevalence in People with Spinal Cord Injury: Age-Period-Duration Effects. Archives of Physical Medicine and Rehabilitation, 86, 1208-1213.

http://dx.doi.org/10.1016/j.apmr.2004.12.023

[6] Guihan, M., Bombardier, C.H., Ehde, D.M., Rapacki, L.M., Rogers, T.J., Bates-Jensen, B., et al. (2014) Comparing Multicomponent Interventions to Improve Skin Care Behaviors and Prevent Recurrence in Veterans Hospitalized for Severe Pressure Ulcers. Archives of Physical Medicine and Rehabilitation, 95, 1246-1253.

http://dx.doi.org/10.1016/j.apmr.2014.01.012

[7] Houghton, P.E., Campbell, K.E., Fraser, C.H., Harris, C., Keast, D.H., Potter, P.J., et al. (2014) Electrical Stimulation Therapy Increases Rate of Healing of Pressure Ulcers in Community-Dwelling People With Spinal Cord Injury. Archives of Physical Medicine and Rehabilitation, 91, 669-678. http://dx.doi.org/10.1016/j.apmr.2009.12.026

[8] Wolff, J. (1986) The Low of Bone Remodeling. Springer, Berlin Heidelberg and New York (Translation of the German 1892 Edition).

[9] Brown, M.D. and Hudlicka, O. (2003) Modulation of Physiological Angiogenesis in Skeletal Muscle by Mechanical Forces: Involvement of VEGF and Mettaloproteinases. Angiogenesis, 6, 1-14.

http://dx.doi.org/10.1023/A:1025809808697

[10] Xiao, D., Wu, S. and Mak, A. (2014) Accumulation of Loading Damage and Unloading Reperfusion Injury-Modeling of the Propagation of Deep Tissue Ulcers. Journal of Biomechanics, 47, 1658-1664. http://dx.doi.org/10.1016/j.jbiomech.2014.02.036

[11] Hatoko, M., Tanaka, A., Kuwahara, M., Yurugi, S., Iioka, H. and Niitsuma, K. (2002) Difference of Molecular Response to Ischemia-Reperfusion of Rat Skeletal Muscle as a Function of Ischemic Time: Study of the Expression of p53, p21 (WAF-1), Bax Protein, and Apoptosis. Annals of Plastic Surgery, 48, 68-74. http://dx.doi.org/10.1097/00000637-200201000-00010

[12] Pietramaggiori, G., Liu, P., Scherer, S.S., Kaipainen, A., Prsa, M.J., Mayer, H., Newalder, J.M., Alperovich, M., Mentzer, S.J., Konerding, M.A., Huang, S., Ingber, D.E. and Orgill, D.P. (2007) Tensile Forces Stimulate Vascular Remodeling and Epidermal Cell Proliferation in Living Skin. Annals of Surgery, 246, 896-902. http://dx.doi.org/10.1097/SLA.0b013e3180caa47f

[13] Kloth, L.C. and Feedar, J.A. (1988) Acceleration of Wound Healing with High Voltage, Monophasic, Pulsed Current. Physical Therapy, 68, 503-508.

[14] Centers for Medicare and Medicaid Services (2002) Coverage and Billing Requirements for Electrical Stimulation for Treatment of Wounds. CMS-Pub.60AB.

[15] Weiss, D., Kirsner, R. and Eaglstein, W. (1990) Electrical Stimulation and Wound Healing. Archives of Dermatology, 126, 222-225. http://dx.doi.org/10.1001/archderm.1990.01670260092018

[16] Lampe, K. (1998) Electrotherapy in Tissue Repair. Journal of Hand Therapy, 11, 131-139. http://dx.doi.org/10.1016/S0894-1130(98)80011-2

[17] Kincaid, C. and Lavoie, K. (1989) Inhibition of Bacterial Growth in Vitro Following Stimulation with High Voltage, Monophasic, Pulsed Current. Physical Therapy, 69, 651-655.

[18] Bourguignon, G. and Bourguignon, L. (1987) Electric Stimulation of Protein and DNA Synthesis in Human Fibroblasts. The FASEB Journal, 1, 398-402.

[19] Rouabhia, M., Park, H., Meng, S., Derbali, H. and Zhang, Z. (2013) Electrical Stimulation Promotes Wound Healing by Enhancing Dermal Fibroblast Activity and Promoting Myofibroblast Transdifferentiation. PLoS ONE, 8, e71660. http://dx.doi.org/10.1371/journal.pone.0071660

[20] Guo, A., Song, B., Reid, B., Gu, Y., Forrester, J.V., Jahoda, C.A.B. and Zhao, M. (2010) Effects of Physiological Electric Fields on Migration of Human Dermal Fibroblasts. Journal of Investigative Dermatology, 130, 2320-2327.

http://dx.doi.org/10.1038/jid.2010.96 
[21] Kawasaki, L., Mushahwar, V.K., Ho, C., Dukelow, S.P., Chan, L.L.H. and Chan, K.M. (2014) The Mechanisms and Evidence of Efficacy of Electrical Stimulation for Healing of Pressure Ulcer: A Systematic Review. Wound Repair and Regeneration, 22, 161-173. http://dx.doi.org/10.1111/wrr.12134

[22] Filius, A., Damen, T.H.C., Schuijer-Maaskant, K.P., Polinder, S., Hovius, S.E.R. and Walbeehm, E.T. (2013) Cost Analysis of Surgically Treated Pressure Sores Stage III and IV. Journal of Plastic, Reconstructive and Aesthetic Surgery, 66, 1580-1586. http://dx.doi.org/10.1016/j.bjps.2013.05.014

[23] Chan, B.C., Nanwa, N., Mittmann, N., Bryant, D., Coyte, P.C. and Houghton, P.E. (2013) The Average Cost of Pressure Ulcer Management in a Community Dwelling Spinal Cord Injury Population. International Wound Journal, 10, 431-440. http://dx.doi.org/10.1111/j.1742-481X.2012.01002.x

[24] Brem, H., Maggi, J., Nierman, D., Rolnitzky, L., Bell, D., Rennert, R., Golinko, M., Yan, A., Lyder, C. and Vladeck, B. (2010) High Cost of Stage IV Pressure Ulcers. The American Journal of Surgery, 200, 473-477. http://dx.doi.org/10.1016/j.amjsurg.2009.12.021

[25] Coyle, P.V., McCaughey, C., Nager, A., McKenna, J., O’Neill, H., Feeney, S.A., Fairley, D., Watt, A., Cox, C. and Curran, T. (2012) Rising Incidence of Pneumocystis Jirovecii Pneumonia Suggests Iatrogenic Exposure of ImmuneCompromised Patients May Be Becoming a Significant Problem. Journal of Medical Microbiology, 61, 1009-1015. http://dx.doi.org/10.1099/jmm.0.043984-0 